21st Particles and Nuclei International Conference (PANIC 2017)

International Journal of Modern Physics: Conference Series

Vol. 46 (2018) 1860035 (6 pages)

(C) The Author(s)

DOI: $10.1142 / \mathrm{S} 2010194518600352$

\title{
Hidden-Charm Decays: An Elegant Probe for Internal Structure
}

\author{
Muhammad Naeem Anwar* \\ CAS Key Laboratory of Theoretical Physics, Institute of Theoretical Physics, \\ Chinese Academy of Sciences, Beijing 100190, China \\ University of Chinese Academy of Sciences, Beijing 100049, China \\ naeem@itp.ac.cn \\ Bing-Song Zou \\ CAS Key Laboratory of Theoretical Physics, Institute of Theoretical Physics, \\ Chinese Academy of Sciences, Beijing 100190, China \\ University of Chinese Academy of Sciences, Beijing 100049, China \\ zoubs@itp.ac.cn
}

Published 3 May 2018

\begin{abstract}
Hadronic transitions are important decay modes of heavy quarkonia and serve as an elegant probe for the internal structure. Several remarkable discoveries have been made to identify the conventional heavy quarkonia and to extract useful information about the so called " $X Y Z$ " exotic states. We studied the hadronic transitions of higher charmonia in the framework of our recently proposed effective model to create light meson(s) in heavy quarkonium transitions. In this contribution, we discuss our results for the charmonium$\eta$ decays of higher $S$ and $D$ wave vector charmonia. We also discuss quantum number $\left({ }^{2 S+1} L_{J}\right)$ assignments for few experimentally observed charmoniumlike states.
\end{abstract}

Keywords: Hadronic Transitions; Higher Charmonia; Effective Model.

\section{Introduction}

Heavy hadron spectroscopy has celebrated almost four decades since the discovery of $J / \psi$ in 1974, so called the "November Revolution". During this era, many theoretical studies have been carried out to produce the spectrum and decays of heavy quarkonia. An important manifestation of studying heavy $Q \bar{Q}$ systems is that it can be well-described by using nonrelativistic formalisms.

In heavy $Q \bar{Q}$ systems, hadronic transitions serve as a crucial probe of their internal structures and help to establish the understanding of light quark coupling

\section{* Speaker}

This is an Open Access article published by World Scientific Publishing Company. It is distributed under the terms of the Creative Commons Attribution 4.0 (CC-BY) License. Further distribution of this work is permitted, provided the original work is properly cited. 
with a heavy degree of freedom. The typical form of the hadronic transitions is

$$
\Phi_{I} \rightarrow \Phi_{F}+h
$$

where $\Phi_{I}$ and $\Phi_{F}$ represent the initial and final quarkonium, respectively; and $h$ stands for the emitted light hadron(s). For lower charmonia, the mass difference $M_{\Phi_{I}}-M_{\Phi_{F}}$ is around a few hundred $\mathrm{MeV}$, consequently the typical momentum of the light hadron(s) is small. Hence, the perturbative Quantum Chromodynamics (QCD) does not work here, nonperturbative approaches are thus needed for studying such hadronic transitions.

In QCD, the well-established formalism for hadronic transitions is multipole expansion $(\mathrm{ME})^{1-3}$, which assumes that the transitions take place due to the intermediate process of gluon emission. These gluons are supposed to be soft, having wavelengths much larger than the size of a heavy quarkonium. These soft gluons further couple to light hadron(s) to complete such kinds of hadronic transitions.

Due to the assumption that the intermediate gluons are soft, the emitted light hadron(s) are predominately of the lower momenta. It is very hard to incorporates the transitions of much higher quarkonia in the framework of QCDME, where we have large decay momenta. For detail discussions and applications of QCDME, we refer the following comprehensive review ${ }^{4}$.

\section{Effective Theories for Hadronic Transitions}

Effective filed theory (EFT) based formalisms have been developed to describe the hadronic transitions. Heavy meson chiral Lagrangians (HMCL) ${ }^{5}$ is the foremost simplification to QCDME. HMCL serve as an EFT to QCDME in a soft exchange where the gluonic exchanges are predominantly of limited momenta. With the assumptions that (i) the heavy $Q \bar{Q}$ involved in the process is well separated to consider it in a stringlike picture and (ii) the momentum of the emitted light meson is not too large, the HMCL are successful at reproducing the hadronic transitions among lower charmonia.

Hadronic transition might occur through intermediate meson loop contributions. The initial heavy $Q \bar{Q}$ can couple to intermediate charmed mesons through the nonperturbative creation of light $q \bar{q}$. This formalism is referred as coupled-channel effects (CCEs). CCEs have been taken into account in the QCDME framework ${ }^{6,7}$. To investigate the intermediate charmed meson loop effects on $\psi^{\prime} \rightarrow J / \psi \eta\left(\pi^{0}\right)$ decay, a nonrelativistic effective field theory (NREFT) formalism was constructed and found that the intermediate loop contribution are sizeable ${ }^{8,9}$.

It is noted that if we go to much higher waves e.g., $\psi(n S)$ or $\psi((n-1) D)$ with $n=4,5,6, \ldots$, the decay momentum is not so small, as it lies in the relativistic regime; hence, the NREFT formalisms are not very suitable for studying hadronic transitions of higher charmonia. The experimental status of the spectrum of higher vector charmonium(like) states is very rich now and several precise measurements have been recorded for their hadronic transitions ${ }^{10}$. To describe these observed 
transitions there is a potential need for a theoretical model which can produce the transitions in the high momentum regime. We try to fulfill this need by modeling hadronic transitions of higher vector charmonia. Our proposed model is away from all the assumptions of HMCL and QCDME, and useful to predict the transitions involving much large momenta.

\section{Our Effective Model and Results}

The effective Lagrangian describing the coupling of a light scalar and pseudoscalar meson with the heavy quark in our model can be descried as

$$
\mathcal{L}_{I}=g\left(\bar{\psi} \psi<\sigma>+\bar{\psi} i \gamma^{5} \psi<\eta>\right),
$$

where $g$ is the overall coupling strength, $\psi$ is the heavy quark field, and $\langle\sigma\rangle$ and $\langle\eta\rangle$ are $S U(3)$ singlet scalar and pseudoscalar meson, respectively. The Lagrangian $\mathcal{L}_{I}$ allows the coupling of the (anti)quark line only to a scalar or isospin singlet pseudoscalar. The possible Feynman diagram for the process $\Psi \rightarrow J / \psi \eta$ (where $\Psi$ is $S$ or $D$ wave higher vector charmonia) is shown in Fig. 1. The Lagrangian $\mathcal{L}_{I}$ of Eq. (2) contains both the scalar and pseudoscalar interactions as present in the Nambu-Jona-Lasinio (NJL) model. ${ }^{19,20}$

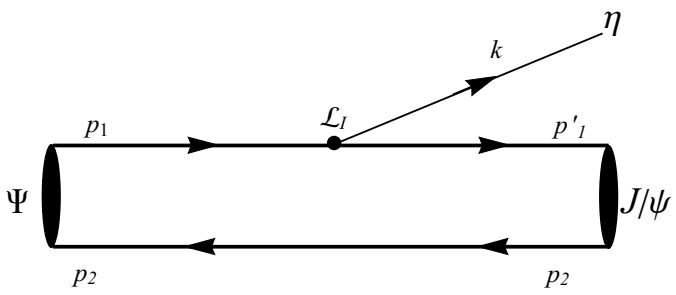

Fig. 1. Quark level description of higher vector charmonia decaying into $J / \psi \eta$.

If we use above Lagrangian to describe the decay process $\Psi \rightarrow J / \psi \eta$, the corresponding decay width and transition amplitude is given as

$$
\Gamma_{A \rightarrow B C}=2 \pi k \frac{E_{B} E_{C}}{m_{A}} \sum_{m_{J_{B}}, m_{J_{C}}} \int d \Omega_{B} \mid \mathcal{M}^{\left.m_{J_{A}} m_{J_{B}} m_{J_{C}}\right|^{2}},
$$

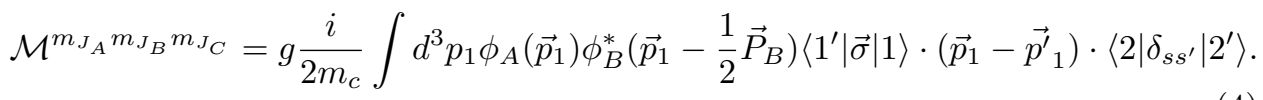

with $m_{c}$ is the mass of charm quark; $E_{B}=\sqrt{m_{B}^{2}+k^{2}}, E_{C}=\sqrt{m_{C}^{2}+k^{2}}$, and $k=\sqrt{\left[m_{A}^{2}-\left(m_{B}-m_{C}\right)^{2}\right]\left[m_{A}^{2}-\left(m_{B}+m_{C}\right)^{2}\right]} / 2 m_{A}$; To evaluate the transition amplitude we use simple harmonic oscillator (SHO) wavefunctions. The model parameters are given in Table 1. 


$$
m_{c}=1.5 \mathrm{GeV} \quad \beta=0.40 \mathrm{GeV} \quad g=0.80 \quad|\theta|=13 \circ
$$

We consider the standard $S-D$ between $S$ and $D$ wave $J^{P C}=1^{--}$charmonia by adopting the well-established formalism based on reproducing the dielectric decay widths to deduce the mixing angle. ${ }^{11,12}$ For an idea of the parameter dependence of our model, we suggest interested readers to see our parametric plots. ${ }^{13}$

\section{1. $\Gamma(\Psi \rightarrow J / \psi \eta)$ and $\Gamma\left(\Psi \rightarrow h_{c} \eta\right)$}

Our results for $\Gamma(\Psi \rightarrow J / \psi \eta)$ are shown in Fig. 2 where we make a comparison with the experimental data. We fit coupling $g$ from the decay process $\psi^{\prime} \rightarrow J / \psi \eta$ and use it to reproduce the decays of next excited charmonia. There exist only upper limits for the $\psi(4160) \rightarrow J / \psi \eta$ and $\psi(4415) \rightarrow J / \psi \eta$ decay processes, our computed decay width for these processes lies within these limits. For the latter process our predicted width is slighter larger than the central value. It is worthy noting that the experimental value of $\Gamma(\psi(4415) \rightarrow J / \psi \eta)$ has large statistical errors. Considering this error range, our prediction in this case still lies within the upper limit.

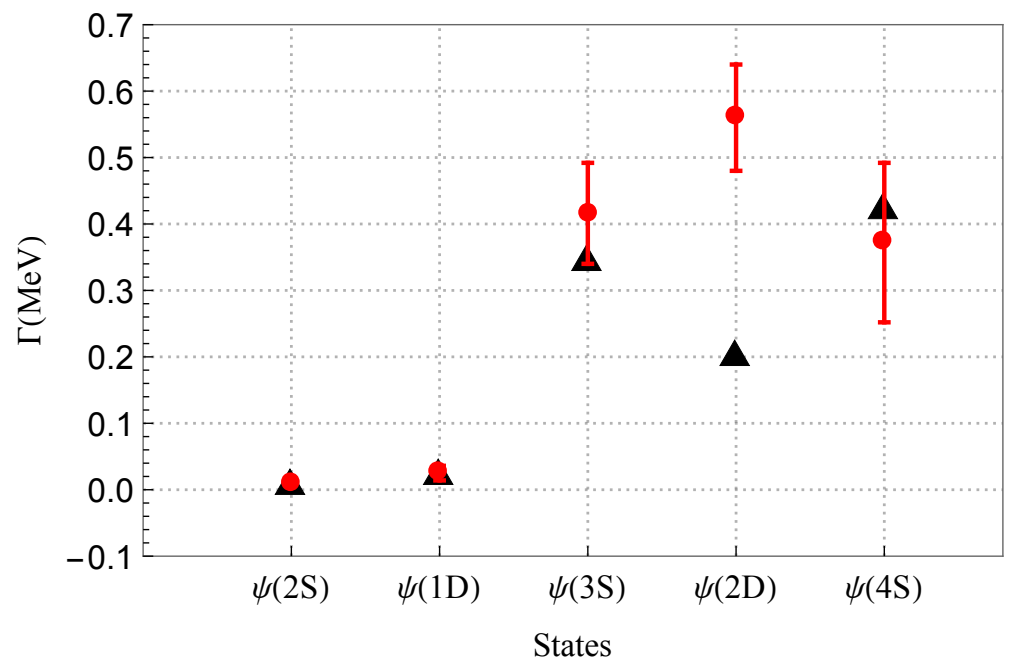

Fig. 2. Decay width of different higher vector charmonium to $J / \psi \eta$. Red filled circles with error bars are the experimental values taken from PDG and the black triangles are our theoretical predictions. For the case of $\psi(2 D)$ and $\psi(4 S)$ we only have the upper limits.

For the decay $\Psi \rightarrow h_{c}(1 P) \eta$, the threshold is $4073 \mathrm{MeV}$. The first state which can decay into $h_{c} \eta$ final state is $\psi(4160)$. The transition $\psi \rightarrow h_{c}(1 P) \eta$ violates heavy quark spin symmetry (HQSS) and expected to be significantly suppressed. ${ }^{14}$ It has been argued in that the CCEs are quite small for this transition. ${ }^{15}$ We predict decay 
width for $\psi(4415) \rightarrow h_{c}(1 P) \eta$ as of the same order of magnitude as $\psi(4160) \rightarrow$ $h_{c}(1 P) \eta$ :

$$
\frac{\Gamma\left(\psi(4160) \rightarrow h_{c}(1 P) \eta\right)}{\Gamma(\psi(4160) \rightarrow J / \psi \eta)}=7.887 \times 10^{-2}, \quad \frac{\Gamma\left(\psi(4415) \rightarrow h_{c}(1 P) \eta\right)}{\Gamma(\psi(4415) \rightarrow J / \psi \eta)}=6.736 \times 10^{-2}
$$

We give estimates of hadronic transition of $\psi(n S /(n-1) D)$ with $(n=4,5,6)$ into $J / \psi \eta$ and $h_{c}(1 P) \eta^{13}$. These states are experimentally unknown, we hope our results provide useful references to search for these sates in experiments.

\section{2. $Y \rightarrow J / \psi \eta$ and $Y \rightarrow h_{c} \eta$}

There are several experimentally observed charmoniumlike vector states which do not decay into charmed mesons, their hidden-charm strong decays would be worth interesting to study. Hence, we consider $Y(4360), Y(4390)$, and $Y(4660)$ and give partial decay widths for $\Gamma(Y \rightarrow J / \psi \eta)$ by assuming them as $c \bar{c}$ bound states with quantum numbers $3^{3} D_{1}, 3^{3} D_{1}$ and $5^{3} S_{1}$, respectively. We only have experimental upper limits ${ }^{17}$ for the product of the branching fraction $\mathcal{B}(Y \rightarrow J / \psi \eta)$ and $\Gamma_{e^{+} e^{-}}(Y)$ for $Y(4360)$ and $Y(4660)$. So one need to use $\Gamma_{e^{+} e^{-}}(Y)$ to get the absolute value for the branching fraction, and we take the average value of $\Gamma_{e^{+} e^{-}}(Y)$ of several theoretical predictions.

\begin{tabular}{|c|c|c|c|c|c|c|c|c|}
\hline \multirow[b]{2}{*}{ State } & \multirow[b]{2}{*}{$n^{2 S+1} L_{J}$} & \multirow[b]{2}{*}{$\Gamma_{\text {total }}$} & \multirow[b]{2}{*}{$\mathcal{B}(Y \rightarrow J / \psi \eta)$} & \multicolumn{3}{|c|}{$\overline{\Gamma_{Y \rightarrow J / \psi \eta}^{\mathrm{th}}}$} & \multicolumn{2}{|c|}{$\overline{\Gamma_{Y \rightarrow J / \psi \eta}^{\exp }}$} \\
\hline & & & & $\theta=0 \circ$ & $\theta=13 \circ$ & $\theta=34 \circ$ & $\theta=0 \circ$ & $\theta=34 \circ$ \\
\hline$\overline{Y(4360)}$ & $3^{3} D_{1}$ & $74 \pm 18[10]$ & $\frac{6.8}{\Gamma e+s-}[10]$ & 0.047 & 0.016 & $1.0 \times 10^{-3}$ & $<0.963$ & $<0.799$ \\
\hline$Y(4390)$ & $3^{3} D_{1}$ & $139.5 \pm 16.1[16]$ & - & 0.083 & 0.028 & $1.6 \times 10^{-3}$ & - & - \\
\hline$Y(4660)$ & $5^{3} S_{1}$ & $48 \pm 15[10]$ & $\frac{0.94}{\Gamma_{e}+e^{-}}[10]$ & 0.057 & 0.070 & 0.077 & $<0.046$ & $<0.116$ \\
\hline
\end{tabular}

Our predictions for $Y(4360)$ are in agreement with the available experimental data. We conclude that $Y(4360)$ could be considered as a potential candidate for dominant $3^{3} D_{1}$ charmonium state. The ratio $\frac{\Gamma\left(Y(4360) \rightarrow h_{c} \eta\right)}{\Gamma(Y(4360) \rightarrow J / \psi \eta)}$ can provide a good test for the structure of $Y(4360)$. We expect this ratio as of the same order of magnitude ${ }^{18}$ as already have observed in the case of $\psi(4160) .{ }^{10}$

For $Y(4660)$, our results indicate that it might has a sizeable $4 D$ component. We also present our prediction for $Y(4390)^{16}$ by assigning it $\psi\left(3^{3} D_{1}\right)$. To identify this state, measurements on its hadronic branching fraction are required.

\section{Summary}

Hidden-charm decays of higher vector charmonia can provide an interesting insight to the internal structure and may lead to the observation of unknown higher charmonia. Hence, the ongoing (Belle and BESIII) and forthcoming ( $\overline{\mathrm{P}} \mathrm{ANDA}$ and BelleII) experiments should look for suggested unobserved decay channels to find $J^{P C}=1^{--}$higher charmonia. The $Y(4360)$ is potentially suitable candidate for 
the $3^{3} D_{1}$ charmonium. However, the suggested ratio may provide another test to its internal structure. We hope that our predictions provide useful references to search and better understanding of higher charmonia.

\section{Acknowledgments}

This work is supported by the National Natural Science Foundation of China (NSFC) through funds provided to the Sino-German CRC 110 "Symmetries and the Emergence of Structure in QCD" (NSFC Grant No. 11621131001), and the CAS-TWAS President's Fellowship for International Ph.D. Students.

\section{References}

1. K. Gottfried, Phys. Rev. Lett. 40, 598 (1978).

2. M. B. Voloshin, Nucl. Phys. B 154, 365 (1979).

3. T. M. Yan, Phys. Rev. D 22, 1652 (1980).

4. Y. P. Kuang, Front. Phys. China 1, 19 (2006).

5. R. Casalbuoni, A. Deandrea, N. Di Bartolomeo, R. Gatto, F. Feruglio and G. Nardulli, Phys. Rept. 281, 145 (1997); Phys. Lett. B 302, 95; 309, 163 (1993).

6. G. Z. Li and Y. P. Kuang, Commun. Theor. Phys. 5, 79 (1986).

7. H. Y. Zhou and Y. P. Kuang, Phys. Rev. D 44, 756 (1991).

8. F. K. Guo, C. Hanhart and U. G. Meissner, Phys. Rev. Lett. 103, 082003 (2009); Erratum: Phys. Rev. Lett. 104, 109901 (2010).

9. F. K. Guo, C. Hanhart, G. Li, U. G. Meissner and Q. Zhao, Phys. Rev. D 83, 034013 (2011).

10. C. Patrignani et al. (Particle Data Group), Chin. Phys. C 40, 100001 (2016).

11. Y. B. Ding, D. H. Qin and K. T. Chao, Phys. Rev. D 44, 3562 (1991).

12. J. L. Rosner, Phys. Rev. D 64, 094002 (2001).

13. M. N. Anwar, Y. Lu and B.-S. Zou, Phys. Rev. D 95, 114031 (2017).

14. M. B. Voloshin, Phys. Rev. D 85, 034024 (2012); Prog. Part. Nucl. Phys. 61, 455 (2008).

15. F. K. Guo, C. Hanhart, G. Li, U. G. Meissner and Q. Zhao, Phys. Rev. D 82, 034025 (2010).

16. M. Ablikim et al. (BESIII Collaboration), Phys. Rev. Lett. 118 (2017) 092002.

17. X. L. Wang et al. (Belle Collaboration), Phys. Rev. D 87, 051101 (2013).

18. M. N. Anwar, Y. Lu and B.-S. Zou, PoS (Hadron2017) 100, arXiv:1712.03425 [hep-ph].

19. Y. Nambu and G. Jona-Lasinio, Phys. Rev. 122, 345; 124, 246 (1961).

20. U. Vogl and W. Weise, Prog. Part. Nucl. Phys. 27, 195 (1991);

S. P. Klevansky, Rev. Mod. Phys. 64, 649 (1992). 